\title{
FLAG and Etoposide as Salvage Treatment in Acute Myeloid Leukemia
}

\author{
Nikolaos Papadantonakis \\ Division of Hematology/Oncology, University of Alabama at Birmingham, Birmingham, AL, USA
}

Patients with acute myeloid leukemia (AML) remain at risk of relapse or may not respond to intensive remission induction chemotherapy. Intensive chemotherapy continues to play an important role in the relapsed/refractory setting with different multiagent regimens and is still relevant despite the advent of molecularly targeted therapies.

In this issue of Acta Haematologica, Hanoun et al. report the outcomes of a combination of FLAG (fludarabine, high-dose cytarabine, and G-CSF) and etoposide (FLAG-Eto). The FLAG backbone has been reported for AML and acute lymphoblastic leukemia as well as in combination with idarubicin. The combination of fludarabine and cytarabine was shown to increase the levels of Ara-C $5^{\prime}$ triphosphate in a seminal study [1]. The G-CSF contribution is thought to be exerted by recruiting cells in the proliferative stage. Etoposide affects topoisomerase II and has been reported to act in synergy with cytarabine in vitro [2]. Etoposide has been used in chemotherapy schemas both in the upfront and relapsed AML settings. However, a combination of FLAG and etoposide has not been extensively reported. This combination, as the authors note, may be useful in patients for whom the risk of using anthracycline may be high. Such situations can arise with high prior cumulative anthracycline exposure, or preexisting cardiac conditions such as congestive heart failure with reduced ejection fraction.

$\begin{aligned} & \text { karger@karger.com } \\ & \text { www.karger.com/aha }\end{aligned}$
Karger $\%$

In Hanoun et al., etoposide was added to FLAG at a dose of $150 \mathrm{mg} / \mathrm{m}^{2} /$ day for 3 days. Notably, in a previously published paper by Aldoss et al. [3] etoposide was administered at $100 \mathrm{mg} / \mathrm{m}^{2} /$ day for 5 days. The study by Hanoun et al. includes older patients (up to the age of 71 years), $30 \%$ of whom received FLAG and idarubicin (FLAG-IDA) previously. The patient population included both refractory $(16.75 \%)$ and relapsed patients, with a sizable proportion (44\%) having already undergone allogeneic hemopoietic stem cell transplantation (alloHSCT).

The complete remission (CR) rate was approximately $25 \%$, with a median overall survival of 17.2 months for patients achieving CR. In the particularly challenging population with post-allo-HSCT relapse, FLAG-Eto showed very modest efficacy (75\% did not achieve CR/CR with incomplete hematological recovery [CRi] or a morphologic leukemia-free state). FLAG-Eto displayed diminished efficacy in patients who had received multiple lines of treatment, but it must be noted that several patients had received FLAG-IDA previously. None of the patients who had received 2 previous salvage therapies attained CR/CRi. Nevertheless, for patients achieving CR, the median remission duration extended past 1 year.

The toxicity profile was notable for universal cytopenias and very frequent infections. Despite toxicities, however, a sizable number of patients who responded to

Nikolaos Papadantonakis, MD, $\mathrm{PhD}$

Division of Hematology/Oncology, University of Alabama at Birmingham 1720 2nd Avenue S.

NP2540U Birmingham, AL 35294 (USA)

E-Mail npapadantonakis@gmail.com 
FLAG-Eto were able to proceed with allo-HSCT or a donor lymphocyte infusion.

The study has several limitations including its small number of patients, the majority of whom had intermediate karyotypes based on MRC (Medical Research Council) criteria. Molecular profiling was limited, and it is unknown how many patients had TP53 or other adverse mutations. Minimal residual disease was also not reported to assess the depth of response, especially before alloHSCT. The mortality rate appears to have been higher than in the experience of Aldoss et al. [3], with infections being the main driver, but it is not possible to draw firm conclusions.

One key question is the role of intensive salvage chemotherapy for patients who are candidates for targeted therapies. The ADMIRAL study compared gilteritinib in FLT3-mutated patients with standard-of-care (SOC) regimens including intensive multiagent salvage chemotherapies, such as FLAG-IDA and MEC (mitoxantrone, etoposide, and cytarabine). Gilteritinib outperformed the SOC arm, and the publication detailing the outcomes is eagerly awaited.

There are currently no available data about the prospective comparison of the IDH $1 / 2$ inhibitors versus salvage chemotherapy for patients harboring IDH mutations. Studies addressing this question could be particularly relevant for patients who could proceed expeditiously to allo-HSCT. Another potential salvage regimen is the combination of a hypomethylating agent with venetoclax [4]. The emerging data are promising, and responses have been noted even in patients with adverse prognostic features and several prior lines of treatment.

Since not all patients have molecularly targeted mutations or may not derive any benefit (including those treated with the CD33+ targeting gemtuzumab ozogamicin), questions remain. What is the optimal salvage intensive chemotherapy regimen? Is there a backbone associated with better outcomes? Are 3 chemotherapy drugs better than 2 in the salvage regimen? None of these issues has been addressed vigorously enough in clinical trials. The outcomes of the Polish Adult Leukemia Group (PALG) study, comparing FLAG-IDA with CLAG-M (cladribine, cytarabine, G-CSF, and mitoxantrone) will be useful (PALG-AML1/2016). Regarding the number of chemotherapy drugs, clinicians must balance the potential for greater toxicity with the improved efficacy of non-crossresistant drugs.

\section{Disclosure Statement}

Dr. Papadantonakis received honoraria and participated on an advisory board of Agios Pharmaceuticals.

\section{References}

1 Gandhi V, Estey E, Keating MJ, Plunkett W. Fludarabine potentiates metabolism of cytarabine in patients with acute myelogenous leukemia during therapy. J Clin Oncol. 1993 Jan;11(1):116-24.

2 Archimbaud E, Leblond V, Michallet M, Cordonnier C, Fenaux P, Travade P, et al. Intensive sequential chemotherapy with mitoxantrone and continuous infusion etoposide and cytarabine for previously treated acute myelogenous leukemia. Blood. 1991 May;77(9): 1894-900.
3 Aldoss I, Ji L, Haider M, Pullarkat V. The combination of fludarabine, cytarabine and etoposide is an active and well-tolerated regimen in relapsed/refractory acute myeloid leukemia. Acta Haematol. 2014;131(4):202-7.

4 Aldoss I, Yang D, Pillai R, Sanchez JF, Mei M, Aribi A, et al. Association of leukemia genetics with response to venetoclax and hypomethylating agents in relapsed/refractory acute myeloid leukemia. Am J Hematol. 2019 Oct;94(10):E253-5. 